members of the patient's direct household who were of North African origin but resident in France remained negative. Microbiologic investigations of a potential origin of the infection in our institution also yielded negative results.

After this unusual pacemaker infection, we investigated further the hypothesis of a relatively immune-depressed background in this patient, who had undergone partial resection of the thymus during cardiac surgery in the neonatal period. The lymphocyte immunophenotype showed a normal profile, and an analysis of interleukin 2 and interferon gamma did not reveal an immune-depressed context. $\mathrm{Hu}-$ man immunodeficiency virus serologic results remained negative. Extensive investigation did not reveal other infected sites.

A quadruple treatment with rifampin (INN: rifampicin), isoniazid, ethambutol, and pyrazinamide for 2 months was followed by double therapy (isoniazid and rifampin) for another 7 months. The strain was susceptible to the 4 antituberculosis drugs. The patient has had good clinical progress with the antituberculosis treatment, but the implantation of a new pacemaker was delayed for several months to prevent recurrence of the infection.

\section{Discussion}

Recurrent and chronic infection of a pacemaker site is a frequent complication once a first infection has occurred. The incidence is higher in the pediatric population and increases with the number of changes of the pacemaker. ${ }^{1}$ Complete surgical removal of the pacing material is therefore considered to be the most effective treatment. ${ }^{1-3}$

Identification of the infectious agent is crucial to target the antibiotic treatment. In $40 \%$ of infected patients, staphylococci are detected in bacteriologic cultures, whereas in $40 \%$ of surgically removed pacing leads, no infectious agent is found. ${ }^{2,3}$ Pacemaker infections by mycobacterial species other than $M$ tuberculosis are rare but well documented. ${ }^{4,5}$ To our knowledge, this is the first case of a pacemaker infection with $M$ tuberculosis. The source may have been among the visitors from the North African origin of the family, where tuberculosis is endemic, but we were unable to investigate. Our case report emphasizes the importance of considering mycobacteria in pacemaker infections, particularly when Gram staining or standard cultures yield negative results.

However rare, pacemaker infection with $M$ tuberculosis must be considered in a patient with a chronic infection, particularly when Gram stain or standard culture results are negative. This is especially true for patients with a family background from a high-burden country.

\section{References}

1. Cohen MI, Bush DM, Gaynor JW, Vetter VL, Tanel RE, Rhodes LA. Pediatric pacemaker infections: twenty years of experience. $J$ Thorac Cardiovasc Surg. 2002;124:821-7.

2. Darouiche RO. Treatment of infections associated with surgical implants. N Engl J Med. 2004;350:1422-9.

3. Baddour LM, Bettmann MA, Bolger AF, Epstein AE, Ferrieri P, Gerber MA, et al. Nonvalvular cardiovascular device-related infections. Circulation. 2003;108:2015-31.

4. Kessler AT, Kourtis AP. Mycobacterium abscessus as a cause of pacemaker infection. Med Sci Monit. 2004;10:CS60-2.

5. Sharma S, Tleyjeh IM, Espinosa RE, Costello BA, Baddour LM. Pacemaker infection due to Mycobacterium fortuitum. Scand J Infect Dis. 2005;37:66-7.

\title{
Intractable postoperative internal thoracic artery spasm managed with angiographic intraluminal papaverine
}

\author{
Lucas H. A. Sanders, FCS(SA), MD, and Mark A. J. Newman, FRACS, Perth, Australia
}

pasm of the left internal thoracic artery (LITA) and native coronary artery in the immediate postoperative period represents a life-threatening complication. Successful treatment with papaverine injected intraluminally by angiographic catheterization has not previously been reported. We present such a case.

\footnotetext{
From Sir Charles Gairdner Hospital (SCGH, Perth, Australia), Department of Cardiothoracic Surgery, Perth, Australia.

Received for publication March 21, 2005; accepted for publication April 5, 2005.

Address for reprints: Lucas H. A. Sanders FCS(SA), MD, Sir Charles Gairdner Hospital (SCGH, Perth, Australia), Department of Cardiothoracic Surgery, Hospital Ave, Nedlands, Perth, WA6007, Australia (E-mail: lucmedi@hotmail.com).

J Thorac Cardiovasc Surg 2005;130:938-40

$0022-5223 / \$ 30.00$

Copyright $\odot 2005$ by The American Association for Thoracic Surgery

doi:10.1016/j.jtcvs.2005.04.022
}

\section{Clinical Summary}

An 84-year-old woman underwent sequential grafting of the first diagonal and left anterior descending coronary artery (LAD) with a pedicled, nonskeletonized left internal thoracic artery (LITA). The procedure was performed without the aid of cardiopulmonary bypass with an Octopus IV Tissue Stabilizer (Medtronic, Inc, Minneapolis, Minn). Preoperative angiography revealed sequential $50 \%$ and $70 \% \mathrm{LAD}$ and $90 \%$ first diagonal stenoses. The right coronary artery was blocked with a small distal vessel to an inferior infarct, well collateralized from the left. Left ventricular function was mildly impaired because of inferior akinesia. Interestingly, left ventriculography revealed hypercontractility in its midportion, resulting in a double-chamber-like image. The patient's history included hypertension, hypercholesterolemia, and peptic ulcer disease. She had stopped smoking 10 years previously. Medications included atenolol, pantoprazole, isosorbide mononitrate, indapamide, ramipril, lercanidipine, pravastatin, nicorandil and clopidogrel (discontinued 3 days before the operation). 


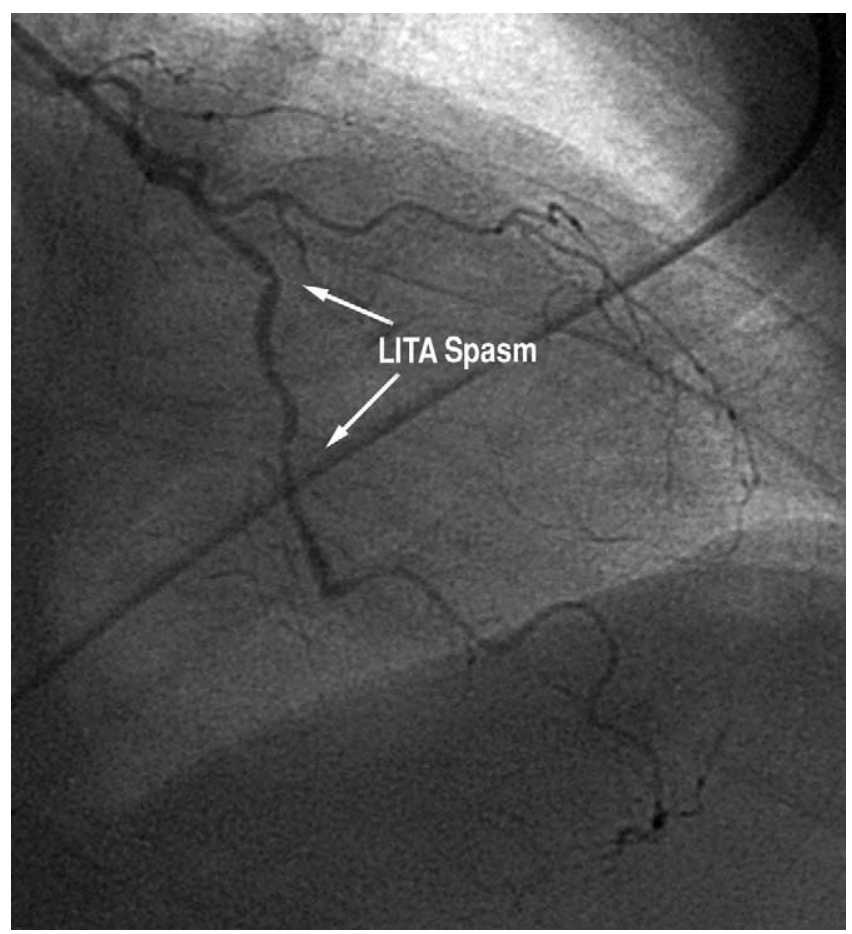

Figure 1. Pretreatment angiography shows areas of spasm in the LITA.

At surgery the mid-LAD portion was intramuscular. After dissection, both the distal LITA and LAD were noted to be in spasm. Papaverine was applied topically (20 mL of 3:1 normal saline solution sprayed with a 26-gauge needle) and intraluminally (20-gauge cannula), resulting in good blood flow from the LITA. The LITA was wrapped in a papaverine-soaked gauze until it was prepared for anastomosis. The operation was performed without incident, and hemodynamics and electrocardiogram were initially normal.

Ventricular fibrillation developed 15 minutes after the patient's arrival in the intensive care unit. After 60 minutes, the patient was stabilized with high doses of epinephrine to maintain arterial blood pressure. Electrocardiography showed signs of inferior ischemia. The patient was transferred to the angiography suite.

Emergency coronary and LITA angiography revealed severe spasm in the LITA segment between the anastomoses to the diagonal and to the LAD (Figure 1). Glyceryl trinitrate injected intraluminally into the LITA had no effect. In contrast, direct angiographic catheter injection of papaverine ( $3 \mathrm{mg} / \mathrm{mL}$ normal saline solution) into the LITA lumen resulted in relief of spasm and significant dilatation of the LITA (Figure 2) as well as the native coronary arteries both distal and proximal to the LITA anastomoses. Epinephrine requirement could be reduced immediately. Amlodipine was commenced. The postoperative course was complicated by atrial fibrillation, pleural effusion, confusion, and hallucinations. The patient was discharged home, in sinus rhythm, 11 days postoperatively with a regimen of amlodipine and amiodarone. Five weeks postoperatively, she appeared well and without angina.

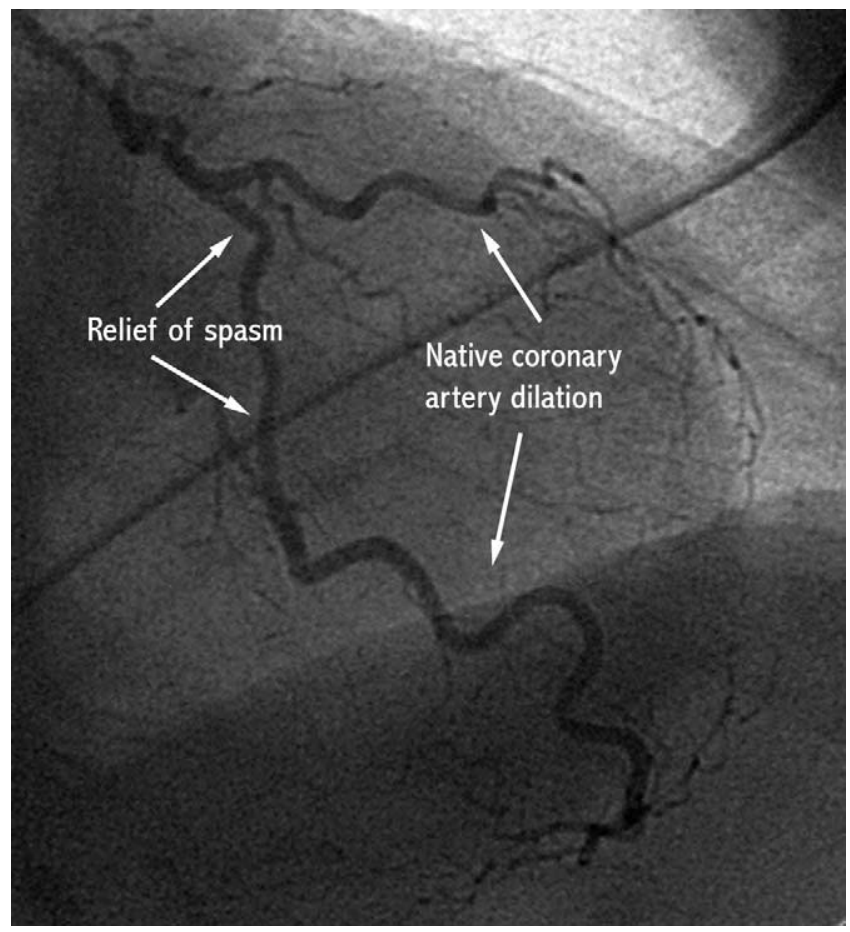

Figure 2. Posttreatment angiography shows relief of LITA spasm and dilatation of native coronary arteries.

\section{Discussion}

Reports of spasm of the LITA and native coronary artery in the immediate postoperative period are marked by an intractable and lethal course. ${ }^{1-3}$ Severe LITA spasm presents with acute hemodynamic collapse, anatomic segmental ST-segment elevation, and ventricular fibrillation.

The mechanism of perioperative vascular spasm is multifactorial. Previous reports have implicated local manipulation, ${ }^{1}$ $\alpha$-adrenergic activity, ${ }^{1}$ high blood $\mathrm{pH},{ }^{1}$ low body temperature, ${ }^{1}$ increased platelet release of vasoconstrictive factors, ${ }^{1}$ increased vasopressin levels, ${ }^{1}$ histamine release, ${ }^{1}$ local increased potassium levels, ${ }^{1}$ possible rebound vasoconstriction after abrupt withdrawal of calcium-channel blockade, ${ }^{1}$ low $\mathrm{PaCO}_{2},{ }^{4}$ smoking, ${ }^{4}$ and use of the distal section of LITA. ${ }^{5}$ Measures that aid in the prevention of postoperative spasm are continued intraoperative use of calciumchannel blockers ${ }^{4}$ and intraoperative local application of vasodilators (nitroglycerin ${ }^{4}$ and papaverine).

Management of postoperative LITA or native coronary artery spasm is challenging. Causative factors should be corrected where possible. The experience with our case led us to believe that a high arterial blood pressure must be maintained for LITA and coronary perfusion. Which inotropic or vasoconstrictive agent is best suited has not been determined. Epinephrine was used successfully in our case. In the event of acute refractory hemodynamic collapse in the immediate postoperative period, Sarabu and colleagues ${ }^{1}$ have recommended immediate reopening. Spasm can then be treated with topical papaverine and additional grafting. Sarabu and colleagues ${ }^{1}$ and Paterson and associates ${ }^{2}$ have suggested infusion of intralu- 
minal vasodilators for postoperative vasospasm (LITA and native coronary spasm). Our patient was successfully treated with papaverine injected intraluminally through angiographic catheterization. This has not been reported before. In our search of the English literature, only one case report of internal thoracic artery spasm refractory to angiographic intraluminal injection with nitroglycerin was found. ${ }^{3}$ In our case, intraluminal nitroglycerin was ineffective in relieving the LITA spasm. Papaverine inhibits oxidative phosphorylation but may cause vasodilatation by inhibiting phosphodiesterase, which prevents breakdown of cyclic nucleotides. Angiographic intraluminal papaverine prevents the complications of surgical delay, reoperation, graft manipulation, external graft injection of vasodilators, repeated use of cardiopulmonary bypass, and additional grafting.

\section{References}

1. Sarabu MR, McClung JA, Fass A, Reed GE. Early postoperative spasm in left internal mammary artery bypass grafts. Ann Thorac Surg. 1987; 44:199-200

2. Paterson HS, Jones MW, Baird DK, Hughes CF. Lethal postoperative coronary artery spasm. Ann Thorac Surg. 1998;65:1571-3.

3. Kitamura H, Nakayama K, Kitano T. A case of refractory spasm of multiple coronary arteries and left internal thoracic artery graft following off-pump CABG. Kyobu Geka. 2001;54:1103-6.

4. Yoshida S, Sakuma K, Yokoyama H, Oda K. [Coronary vasospasm during off-pump coronary artery bypass grafting: report of two cases.] Japanese. Kyobu Geka. 2003;56:585-8.

5. He GW. Contractility of the human internal mammary artery at the distal section increases toward the end. Emphasis on not using the end of the internal mammary artery for grafting. J Thorac Cardiovasc Surg. 1993;106:406-11.

\section{Stenosis of the grafted portion of the coronary artery after aortic root replacement with a Freestyle prosthesis}

Masanori Murakami, MD, ${ }^{\text {a }}$ Haruhiko Okada, MD, ${ }^{a}$ Masahiko Nishida, MD, ${ }^{a}$ and Kimikazu Hamano, MD, ${ }^{\text {b }}$ Yamaguchi, Japan

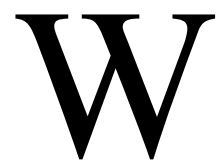
e report a case of stenosis at the grafted portion of the coronary artery after aortic root replacement with a Freestyle prosthesis (Freestyle Aortic Root Bioprosthesis; Medtronic, Inc, Minneapolis, Minn). The coronary artery was reconstructed with the full root technique. There was no displacement or flexion of the coronary artery graft as a result of the surgical procedure. Aspirin was used as a postoperative anticoagulant. A coronary angiogram obtained 18 months after the surgery showed $90 \%$ stenosis at the left coronary ostium (Figure 1) and 75\% stenosis at the right coronary ostium. No stenosis (0\%) was observed after percutaneous coronary intervention (PCI). After PCI, the patient received adequate doses of warfarin sodium and aspirin. The follow-up coronary angiogram (at 18 months) showed no graft stenosis. This is the first

\footnotetext{
From the Division of Cardiovascular Surgery, Shakaihoken Tokuyama Central Hospital, a and the First Department of Surgery, Yamaguchi University School of Medicine, ${ }^{\mathrm{b}}$ Yamaguchi, Japan.

Received for publication April 8, 2005; accepted for publication April 25, 2005.

Address for reprints: Masanori Murakami, MD, Division of Cardiovascular Surgery, Shakaihoken Tokuyama Central Hospital, Kohdachoh 1-1, Shunan, Yamaguchi 745-8522, Japan (E-mail: surg-1@ yamaguchi-u.ac.jp).

J Thorac Cardiovasc Surg 2005;130:940-1

$0022-5223 / \$ 30.00$

Copyright $\odot 2005$ by The American Association for Thoracic Surgery doi:10.1016/j.jtcvs.2005.04.024
}

English-language re-

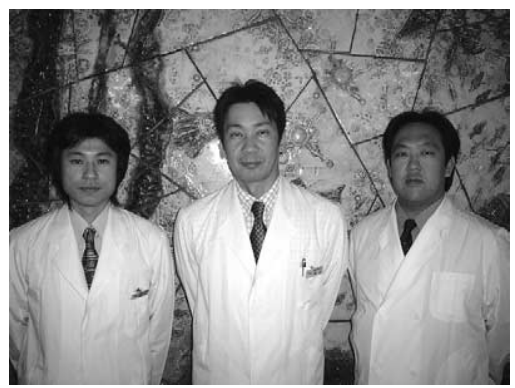
port of PCI performed for coronary graft stenosis after aortic root replacement with a Freestyle prosthesis.

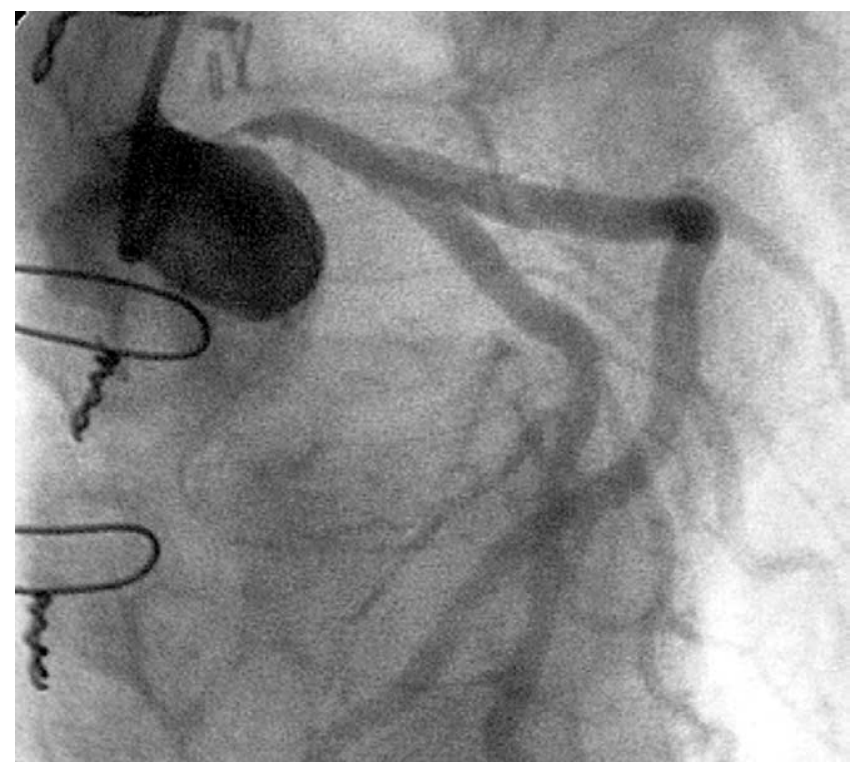

Figure 1. Angiogram of reconstructed left coronary artery showing $\mathbf{9 9 \%}$ stenosis of grafted portion. 